\title{
Liver Test Monitoring: Real-World Compliance for Drugs with Monitoring Requirements at 2-Week Intervals or More Frequently
}

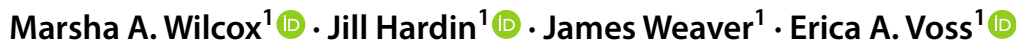

Published online: 8 August 2019

(c) The Author(s) 2019

\begin{abstract}
Background Monitoring risk is often an important component of therapy. Some compounds require liver test (LT) monitoring, with the frequency detailed in the product label. Compliance with these instructions is generally unknown.

Objective The goal of this short study was to describe LT compliance for compounds with monitoring recommended at 2-week intervals or more frequently in three US administrative claims databases.

Methods The sample was drawn from three US claims databases during the period 1 January 2015 through 30 June 2018. This study examined nine compounds and five types of LTs. We looked at compounds in a published list of drugs requiring LTs at 2-week intervals or more frequently. Descriptive statistics about the days between tests were reported, as were the number and proportion of tests associated with each drug that met the recommended frequency.

Results Compliance was $<33 \%$ with four drugs (ketoconazole, succimer, pentamidine, and felbamate) and $>60 \%$ with five drugs (oxaliplatin, rifampin, tolcapone, albendazole, and azathioprine). Among drugs with more than 1000 drug eras observed (all but succimer and tolcapone), LT compliance was highest for oxaliplatin (75.3\%) and lowest for pentamidine (20.6\%), with little difference in overall compliance by type of test (range 41-46).

Conclusion Compliance with frequent LT monitoring differed for the drugs examined. Two strata were found: compliance $>60 \%$ (oxaliplatin, rifampin, tolcapone, albendazole, and azathioprine) and compliance 20-30\% (ketoconazole, succimer, pentamidine, and felbamate). No drug reached $80 \%$ compliance.
\end{abstract}

\section{Key Points}

Compliance with liver test (LT) monitoring requirements for drugs requiring hepatic monitoring at 2-week intervals or more frequently appears to be bimodal, either $60-70 \%$ or $20-30 \%$.

Administrative healthcare data can be useful for examining LT compliance but are subject to contracts with laboratories and limited patient enrollment.

Electronic supplementary material The online version of this article (https://doi.org/10.1007/s40290-019-00294-z) contains supplementary material, which is available to authorized users.

Marsha A. Wilcox

MWilcox@its.jnj.com

1 Janssen Pharmaceutical Research and Development, LLC, 1125 Trenton-Harbourton Rd., Titusville, NJ 08560, USA

\section{Background}

Drug-induced liver injury is a common reason for US FDAapproved compounds to be withdrawn from the market [1]. Monitoring risk is often an important component of therapy. One hallmark case of the importance of liver testing is troglitazone, a thiazolidinedione used in the treatment of type 2 diabetes mellitus introduced in the USA in 1997. Clinical trials reported some indications of adverse liver events, specifically elevated transaminase levels [2]. When the drug was first marketed, the label included no recommendations for liver test (LT) monitoring. As use of the drug increased, cases of acute liver failure became evident. In response, a series of letters to healthcare professionals were issued with increasing recommendations for liver enzyme monitoring. In 2000, troglitazone was removed from the market because of the number of cases of liver failure [2]. Graham et al. [3] showed that transaminase monitoring was "infrequently and irregularly performed", even with repeated letters to healthcare providers [4]. 
On the other hand, not all drug-related LTs are as clinically useful as initially intended. In 2006, a report by the National Lipid Association's Statin Liver Safety Task Force recommended that LTs with statin use be reduced to a prestatin baseline and in cases in which they were clinically indicated for other reasons [5]. Otherwise, follow-up liver enzyme testing was not required after statin initiation. The report was updated in 2014, and the basic findings were the same: regular LT monitoring in patients receiving statins was unnecessary [6].

Dusetzina et al. [7] reviewed the literature on the impact of FDA drug risk communications on medication, healthcare services use, and health outcomes. They concluded that some communications had a strong impact, whereas others had minimal or delayed effects on utilization or health behaviors. The authors suggested continued assessments of advisories and label changes [7].

Some compounds require liver function monitoring, with testing frequency detailed in the product label. Physician compliance with these instructions is generally unknown. Several reports have considered compliance for specific compounds but little has been written about overall compliance rates $[3,10,11]$.

In some cases, compliance with recommended testing changes over time. The risk evaluation and mitigation strategy for bosentan, used in the treatment of pulmonary artery hypertension, includes a requirement of review of LTs before prescribing and monthly testing during therapy [8]. These authors examined claims records spanning more than 12 years and found that compliance was less than perfect, with $70 \%$ of patients having at least one LT. The compliance rate declined for those with 12 or more administrations of the drug. Compliance was $>90 \%$ for only about one in three patients receiving the drug [9]. Lapatinib is prescribed for breast cancer and other solid tumors; the label was changed to add LT monitoring. A claims-based study of physician adherence to recommended LT guidelines showed an increase in LTs after monitoring was added to the label [10]; overall compliance following the addition was just over $80 \%$. In another retrospective cohort study, compliance with recommended LT for patients with tuberculosis in Taiwan was examined in national health records. Newly diagnosed patients were categorized as completely, partially, or non-adherent. Compliance rates differed for patients with a history of liver disease or viral hepatitis or a prescriber with a specialty in chest, tuberculosis, or infectious disease [11].

\section{Objective}

The objective of this study was to describe LT compliance for compounds with monitoring recommended at 2 -week intervals or more frequently in three US claims databases. Compliance is defined by an LT both being ordered by a healthcare professional and administered to a patient.

\section{Methods}

\subsection{Liver Testing Frequency}

Our sampling frame for compounds was a published list of drugs requiring hepatic monitoring [12]. We included compounds requiring monitoring at 2-week intervals or more frequently. The drugs examined in this study and corresponding recommended LT frequencies can be found in Table 1. Ketoconazole is an antifungal agent administered both topically and orally. Azathioprine, an immunosuppressive agent, is administered orally and by injection. Oxaliplatin, given by injection, is used to treat colorectal cancer. Rifampin is used most often to treat tuberculosis and is given orally. Pentamidine, administered via injection or inhalation, is an antimicrobial used to treat several infections, among them pneumocystis pneumonia in immunocompromised patients. Albendazole is used to treat a variety of parasitic worm infestations and is given orally. Felbamate, given orally as tablets or suspension, is an anticonvulsant. Succimer is administered orally to treat lead and other poisoning. Finally, tolcapone is given orally to treat Parkinson's disease. LT frequency was defined by days between LTs during a drug era, a period of inferred persistent drug exposure, during the defined study period (1 January 2015 to 30 June 2018). A drug era began with the first observed dispensing of a prescribed drug in the database and continued through the days supplied. A gap between dispensings of $<30$ days was considered the same era. The observation for a drug used for a finite period reflected the duration of treatment.

\subsection{Patient Sample}

The sample was drawn from three large US administrative claims databases during the period 1 January 2015 through 30 June 2018 , as follows:

1. IBM MarketScan ${ }^{\circledR}$ Commercial Database (CCAE), which contains data from individuals enrolled in US employer-sponsored insurance health plans. The data included adjudicated health insurance claims (e.g., inpatient, outpatient, and outpatient pharmacy) and enrollment data from large employers and health plans who provide private healthcare coverage to employees, their spouses, and dependents. Additionally, laboratory tests are available for a subset of the covered lives. This claims database includes a variety of fee-for-service, preferred provider organizations, and capitated health plans.

2. IBM MarketScan ${ }^{\circledR}$ Multi-State Medicaid Database (MDCD) adjudicated US health insurance claims for 
Table 1 Drugs and recommended liver testing frequency

\begin{tabular}{|c|c|c|c|c|c|c|c|c|}
\hline Name, year introduced & ALT & AST & Bilirubin & ALP & GGT & Hepatic function monitoring requirement & $\begin{array}{l}\text { Days } \\
\text { between } \\
\text { tests }\end{array}$ & Drug era time window \\
\hline Azathioprine, 1957 & $\checkmark$ & $\checkmark$ & $\checkmark$ & $\checkmark$ & & $\begin{array}{l}\text { Every } 2 \text { weeks in the first } 4 \text { weeks; periodi- } \\
\text { cally thereafter }\end{array}$ & 14 & 28 days from first era \\
\hline Ketoconazole, 1977 & $\checkmark$ & $\checkmark$ & $\checkmark$ & $\checkmark$ & & $\begin{array}{l}\text { Before therapy; frequent intervals during } \\
\text { therapy (weekly) }\end{array}$ & 7 & All \\
\hline Pentamidine, 1937 & $\checkmark$ & $\checkmark$ & $\checkmark$ & $\checkmark$ & & $\begin{array}{l}\text { Before, during, and after therapy (twice } \\
\text { weekly) }\end{array}$ & $\sim 3$ & All \\
\hline Albendazole, 1975 & $\checkmark$ & $\checkmark$ & & & & $\begin{array}{l}\text { Beginning of each cycle; at least every } \\
2 \text { weeks during therapy }\end{array}$ & 14 & All \\
\hline Felbamate, 1993 & $\checkmark$ & $\checkmark$ & & & & $\begin{array}{l}\text { Before initiation; } 1 \text { - to 2-week intervals during } \\
\text { therapy }\end{array}$ & 14 & All \\
\hline Rifampin, 1971 & $\checkmark$ & $\checkmark$ & & & & Before therapy; $2-4$ weeks during therapy & 28 & All \\
\hline Succimer, 1991 & $\checkmark$ & $\checkmark$ & & & & Before therapy; weekly thereafter & 7 & All \\
\hline Tolcapone, 2009 & $\checkmark$ & $\checkmark$ & & & & $\begin{array}{l}\text { Every } 2 \text { weeks during the first year; } 4 \text { weekly } \\
\text { for the next } 6 \text { months; } 8 \text { weekly thereafter }\end{array}$ & 14 & 365 days from first era \\
\hline Oxaliplatin, 2002 & $\checkmark$ & $\checkmark$ & $\checkmark$ & & & Before each treatment cycle (every 2 weeks) & 14 & All \\
\hline Pemoline $^{\mathrm{a}}$ & $\checkmark$ & & & & & Baseline, every 2 weeks while on drug & 14 & All \\
\hline Tacrine $^{\mathrm{a}}$ & $\checkmark$ & $\checkmark$ & $\checkmark$ & & $\checkmark$ & $\begin{array}{l}\text { Every other week from at least week } 4 \text { to } 16 \text { of } \\
\text { treatment }\end{array}$ & 14 & 112 days from first era \\
\hline
\end{tabular}

$A L T$ alanine aminotransferase, $A L P$ alkaline phosphatase, $A S T$ aspartate aminotransferase, $G G T$ gamma-glutamyl transpeptidase

${ }^{a}$ Drugs not included in the results either because they were discontinued in the USA or because definition of the drug era was not feasible

Medicaid enrollees from multiple states. It includes hospital discharge diagnoses, outpatient diagnoses and procedures, outpatient pharmacy claims, ethnicity, and Medicare eligibility. Members maintain their same identifier even if they leave the system for a brief period; however, the dataset lacks laboratory results data.

3. Optum $\odot$ Deidentified Clinformatics ${ }^{\circledR}$ Data Mart Database-Socio-Economic Status (SES), which is an adjudicated administrative health claims database for members with private health insurance, who are fully insured in commercial plans or in administrative services only, Legacy Medicare Choice Lives (prior to January 2006), and Medicare Advantage (Medicare Advantage Prescription Drug coverage starting January 2006). The population is primarily representative of US commercial claims patients (aged 0-65 years) with some Medicare (aged $\geq 65$ years), but ages are capped at 90 years. It includes data captured from administrative claims processed from inpatient and outpatient medical services and prescriptions as dispensed, as well as results for outpatient laboratory tests processed by large national laboratory vendors who participate in data exchange with Optum. Optum SES provides socioeconomic status for members with both medical and pharmacy coverage and location information for patients at the US Census Division.

\subsection{Compliance}

Compliance was defined as the number and proportion of intervals in which the recommended frequency of testing was met divided by the total number of intervals with a test for a given drug. Test compliance is measured by observing a record of a test in the database. A test record is observed in the database when a provider orders the test and the patient completes the test. Compliance therefore is attributable to both the provider and the patient.

Since there are some differences between available datasets with respect to years available and patient characteristics, the detailed results were stratified by database. The detailed results can be found in the electronic supplementary material. Figure 1 shows an example drug era (i.e. duration of time it is inferred that a patient is exposed to a particular ingredient).

Most compounds have specific recommended administration windows. For example, the recommended testing for azathioprine is every 2 weeks for the first 4 weeks of administration. Our compliance definition is specific to the first 4 weeks of administration of the drug. Similarly, LTs for tolcapone are recommended every 2 weeks during the first year of administration. Our observation window for that drug ends after 365 days from the first exposure. In all cases, observation begins with the first administration of the drug in the database. 
Fig. 1 Example of a drug with recommended weekly liver testing (LT) monitoring

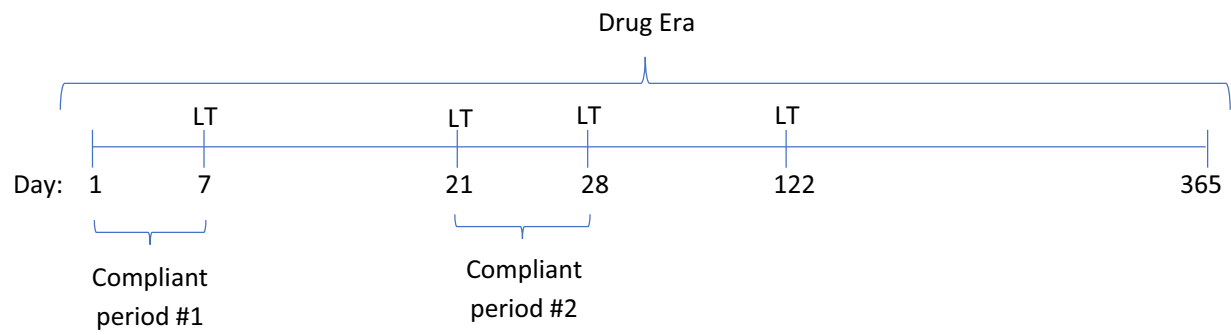

\subsection{Statistics}

Our analyses used descriptive statistics, including the number of patients receiving the drug, the median and mean days between tests, standard deviation of the mean, and the minimum and maximum number of days between tests. Our metrics are the number and proportion of tests associated with each drug that meet the recommended frequency. We also reported testing rates by the individual types of LT.

\section{Results}

The drugs included in these analyses were azathioprine, ketoconazole, pentamidine, albendazole, felbamate, rifampin, succimer, oxaliplatin, and tolcapone. We did not include pemoline or tacrine because they were either discontinued in the USA or the definition of the drug era was not feasible. Table 1 shows which LTs are required and the recommended monitoring interval for each drug.

Compliance rates were bimodal, with compliance rates $<33 \%$ for four drugs (ketoconazole, succimer, pentamidine, and felbamate) and $>60 \%$ for five drugs (oxaliplatin, tolcapone, rifampin, albendazole, and azathioprine) (Table 2). Among drugs with more than 1000 drug eras observed (all but tolcapone and succimer), LT compliance was highest for oxaliplatin (75.3\%) and lowest for pentadine $(20.6 \%)$, with little difference in the overall compliance rate by type of test (range 41-46).

\section{Discussion}

In the three large US claims data sources we used, compliance with frequent LT monitoring differed for the drugs examined. There were two strata: compliance rates $>60 \%$ (oxaliplatin, tolcapone, rifampin, albendazole, and azathioprine) and between about 20 and 30\% (ketoconazole, succimer, pentamidine, and felbamate). No drug met a compliance rate of $80 \%$.

Compliance for drugs used for a finite period ranged widely (e.g., albendazole [66\%], rifampin [67\%], ketoconazole [32\%], succimer [29\%], and pentamidine [21\%]), as it did for drugs with longer typical courses of treatment (Table 2). There was no apparent difference by duration of treatment or length of time on the market. All the drugs we examined had been on the market for at least 10 years.

The objective of this concise report was to examine compliance with recommended LTs in compounds with testing recommended at 2-week intervals or more frequently. It would be interesting to examine factors that might influence compliance, including patient comorbidities, indications, prescriber specialty, and patient demographics.

Understanding the motivations for higher LT compliance rates could potentially lead to the improvement of current programs or the design of new programs to increase LT monitoring where it is warranted for patient safety. The apparent bimodal distribution of compliance with testing is important for consideration in the marketing of new compounds. Not all recommended testing is done and, in some cases, is infrequently done.

This study had some limitations. Of note, LT data were incomplete in the three data sources, and the subset for whom the data were captured is not necessarily representative of the population for whom an LT has been ordered. The healthcare claims databases we used were likely not representative of anything beyond patients in the databases. A non-random subset of laboratories provides test results for inclusion in the databases; the subset of patients for whom values were present in the database was also non-random. As a result, the sample used in these analyses may have differed from the population of patients with LTs. Compliance rates could have been biased in either direction [13].

Some insight into the process by which the databases are assembled might be helpful. Contracts for obtaining diagnostic measurement data were renewed annually. As a result, the sample of patients for whom laboratory measurement data were present varied over time. Patients continually enroll and disenroll from health insurance plans; the average length of time in the databases is generally about 2 years. It is likely we did not have a complete LT history for any given patient. We did, however, use all available data.

Individuals who lack or have insufficient medical insurance were underrepresented. IBM CCAE and Optum data only represented those who were commercially insured. Further, the IBM Medicaid data were only available for 11 states. 
Table 2 Drug era count, compliance, and patient count

\begin{tabular}{lrrrr}
\hline Compound & Drug era count & Number compliant & \% compliant & Patient count \\
\hline Oxaliplatin & 55,940 & 42,103 & 75.26 & 47,699 \\
Rifampin & 33,265 & 22,515 & 67.68 & 30,626 \\
Tolcapone & 116 & 78 & 66.87 & 101 \\
Albendazole & 1685 & 1113 & 66.03 & 1614 \\
Azathioprine & 151,662 & 93,006 & 61.32 & 114,091 \\
Ketoconazole & 423,820 & 136,469 & 32.20 & 370,932 \\
Succimer & 162 & 47 & 29.16 & 139 \\
Felbamate & 1592 & 344 & 21.62 & 1320 \\
Pentamidine & 1702 & 351 & 20.61 & 1183 \\
Test & & & & \\
Bilirubin & 358,434 & 164,351 & 45.85 & 296,584 \\
ALT & 127,422 & 54,862 & 43.06 & 110,537 \\
AST & 90,913 & 37,821 & 41.60 & 79,011 \\
ALP & 83,503 & 34,736 & 41.60 & 72,800 \\
GGT & 9672 & 4255 & 44.00 & 8772 \\
\hline
\end{tabular}

$A L T$ alanine aminotransferase, $A L P$ alkaline phosphatase, AST aspartate aminotransferase, GGT gammaglutamyl transpeptidase

\section{Conclusions}

The objective of this concise report was to examine compliance with recommended LTs in compounds with testing recommended at 2-week intervals or more frequently. We found a bimodal distribution, with compliance in four drugs $<33 \%$ and in five drugs $60-75 \%$. None of the compounds we examined reached $80 \%$. Tracking LT compliance and implementing programs to increase compliance with testing recommendations could improve patient outcomes.

\section{Compliance with Ethical Standards}

Funding This work was funded by Janssen Pharmaceutical R\&D, LLC., a Johnson \& Johnson company. Open access was funded by Janssen Pharmaceutical R\&D, LLC.

Conflict of interest Marsha Wilcox, Jill Hardin, James Weaver, and Erica Voss are employees and shareholders of Johnson \& Johnson.

Ethical Approval The use of the IBM MarketScan ${ }^{\circledR}$ Commercial Database Database was reviewed by the New England Institutional Review Board (IRB) and was determined to be exempt from broad IRB approval as the research project did not involve human subjects.

Open Access This article is distributed under the terms of the Creative Commons Attribution-NonCommercial 4.0 International License (http://creativecommons.org/licenses/by-nc/4.0/), which permits any noncommercial use, distribution, and reproduction in any medium, provided you give appropriate credit to the original author(s) and the source, provide a link to the Creative Commons license, and indicate if changes were made.

\section{References}

1. Ahmad J, Odin J. Epidemiology and genetic risk factors of drug hepatotoxicity. Clin Liver Dis. 2017;21(1):55-72.

2. LiverTox: clinical and research information on drug-induced liver toxicity: Troglitzzone. https://livertox.nih.gov/Troglitazone.htm. Accessed 4 June 2019.

3. Graham DJ, Drinkard CR, Shatin D, et al. Liver enzyme monitoring in patients treated with troglitazone. JAMA. 2001;286:831.

4. Cluxton RJ Jr, Li Z, Heaton PC, et al. Impact of regulatory labeling for troglitazone and rosiglitazone on hepatic enzyme monitoring compliance: findings from the state of Ohio Medicaid program. Pharmacoepidemiol Drug Saf. 2005;14:1-9.

5. McKenney JM, Davidson MH, Jacobson TA, Guyton JR. National lipid association statin safety assessment task force. Final conclusions and recommendations of the National Lipid Association Statin Safety Assessment Task Force. Am J Cardiol. 2006;97(8A):8994C. https://doi.org/10.1016/j.amjcard.2006.02.030.

6. Bays H, Cohen D, Chalasani N, Harrison S. An assessment by the Statin Liver Safety Task Force: 2014 update. J Clin Lipid. 2014;8:S47-57.

7. Dusetzina SB, Higashi AS, Dorsey ER, et al. Impact of FDA drug risk communications on health care utilization and health behaviors: a systematic review. Med Care. 2012;50(6):466-78.

8. Bosentan REMS Program. https://www.bosentanremsprogram. com/BosentanUI/home.u. Accessed 4 June 2019.

9. Blanchette CM, Nunes AP, Lin ND, Mortimer KM, Noone J, Tangirala K, Johnston S, Gutierrez B. Adherence to risk evaluation and mitigation strategies (REMS) requirements for monthly testing of liver function. Drugs Context. 2015;4:212272. https://doi. org/10.7573/dic.212272.

10. Landis SH, Chen C, Byrne JA, Jones SE, Dhanda R, Nelson JJ. Assessment of physician compliance to liver function test monitoring guidance for patients treated with lapatinib. Commun Oncol. 2013;10:258-65.

11. Chou HC, Lin SW, Chen WW, Ke WM, Chao PH, Hsiao FY. Monitoring liver function among patients who initiated antituberculosis drugs in Taiwan, 2000-2011. Int J Tuberc Lung Dis. 2014;18(10):1245-51. https://doi.org/10.5588/ijtld.14.0155. 
12. Tice SA, Parry D. Medications that require hepatic monitoring. Hosp Pharm. 2004;39(6):595-606.

13. Makadia R, Hardin J, Hester L, Knoll C, Londhe A, Swerdel J. Comparabity assessment of cohorts with and without laboratory values. 2017. Poster presented at the OHDSI meeting, Bethesda. http://www.ohdsi.org/web/wiki/lib/exe/fetch.php?media=resou rces:laboratory_compare_makadia_hardin_v10.pdf. Accessed 4 June 2019. 